sections on anatomy and physiology; the evaluation of the dizzy patient - an analysis of history, examination and laboratory tests and the diagnosis and treatment of common neuro-otologic disorders. The last is disappointingly brief and in many ways uncritical, and old fashioned. By contrast, the first two sections are detailed, precise and provide a most helpful current account of clinically relevant anatomy and investigation. MRI, evoked potential techniques, electronystagmography and the newer rotational investigations of semicircular canal function are considered and well illustrated.

I think this a useful book, which attempts and to a large measure succeeds in illuminating a difficult, tenebrous area of neurology. It is not cumbersome in size or content and will certainly commend it to colleagues and junior staff in training.

JMS PEARCE

Neuroendocrinological Aspects of Neurosurgery. Proceedings of the Third Advanced Seminar in Neurosurgical Research, Venice April 30-May 11987. Edited by J D PICKARD, F COHADON, J LOBO Antunes. (Pp 128; Price: DM158.) Wien, Springer-Verlag, 1990. ISBN 3-211-82160-

Under the guidance of its recent and current chairmen, the Research committee of the European Association of Neurological Surgeons, with the support of Fidia, has held a series of very successful meetings, directed at younger staff neurosurgeons and senior trainees with an active involvement in research, designed to link selected areas of clinical neurosurgery with basic neuroscience. This book is the proceedings of the third (1987) meeting.

The first half of the book covers anatomy, physiology, neurohumoral and clinical aspects of the hypothalamo-pituitary axis. Everitt and Hökfelt provide an authoritative description of the anatomy of the hypothalamus and its connections. They illustrate the wealth of information now available on projections, receptors, and neurotransmitter co-existence. An attempt is made to integrate some of this material in the chapter by Vincent and Simonnet which follows. They emphasise the analogies between classical neurotransmission and secretion, and the coupling of behavioural and neuroendocrine homeostatic mechanisms, for example drinking and vasopressin secretion in response to osmotic stimulation. Some newer concepts are also developed, such as dendritic liberation of neurotransmitters, and autoreceptors. The chapters by Page et al and Teasdale $e t$ al are excellent reviews of the medical and surgical aspects of hypothalamic and pituitary diseases and their treatment.

However, it is the group of chapters on the relationships of the CNS with systemic metabolism and fluid balance in subarachnoid haemorrhage and head injury which give this book much broader relevance, for physicians as well as for surgeons. There are excellent and very lucid reviews of CNS control of fluid balance, and its disturbances in neurosurgical patients, by Lightman and Walker respectively. The chapters by NeilDwyer, Wijdicks, Nelson and Doczi and their respective collaborators are essential reading for vascular neurosurgeons and neurologists.
The use of double column A4 format provides for a large volume of information within 128 pages, and results in a conveniently thin book which will slip easily into a briefcase. It is an excellent expression of the collective strengths of European clinica neuroscience, and the price makes it a good buy for a departmental library. Potentia specialty fellowship candidates-and examiners - may well find it useful. ANTHONY STRONG

Conceptual Issues in Psychological Medicine. By MICHAEL SHEPHERD. (Pp 335 Price £40.00) London, Routledge, 1990. ISBN 0-415-03727-1.

The title of this book is misleading. Professor Shepherd has already published two volumes of his collected papers and this third volume contains a motley collection of papers, essays and commentaries that for one reason, or another were unsuitable for inclusion in either of the earlier collections. A hundred pages, no less, are occupied by case histories of morbid jealousy and half the other eighteen articles are brief essays of less than five thousand words. All the same they make compelling bedside reading, for Michael Shepherd is always thoughtful and perceptive with a fluent pen, a well developed sense of history and a keen eye for the shortcomings of contemporary Anglo American orthodoxies. $\mathrm{He}$ is also impressively erudite and, like Macaulay, Aldous Huxley and his mentor Aubrey Lewis, delights in trailing his learning before his readers. The paper on morbid jealousy is, despite its impedimenta, a classical clinical description and there are important messages for contemporary psychiatry in several of the historical and literary pieces.

R E KENDELL

\section{Immunologic Mechanisms in} Neurologic and Psychiatric Disease. (Research Publications: Association for Research in Nervous and Mental Disease, Vol. 68) Edited by BYRON H. WAKSMAN. (Pp 336; Price \$144.00) New York, Raven Press, 1990. ISBN 0-88167-593-8.

This volume admirably maintains the high standard of its numerous predecessors dating from 1920. In view of the large number of books on neuroimmunology which have appeared in recent years, it is always somewhat daunting to have to confront yet another volume on this subject. However, in this case the effort is certainly worthwhile since this book provides a large number of high quality reviews all of which display a uniformly consistent standard of discussion and presentation. The two broad messages which came over to me after reading this were firstly, that basic mechanisms in immunology are being recognised as being increasingly complex in nature which in turn is reflected by the increasing sophistication of the concepts and techniques applied to neuroimmunological problems; and secondly, that the evidence for a close association between the immune systems and the CNS is increasing at a rapid rate.

The book is divided into several sections which follow each other in a logical and coherent sequence. The opening chapters cover basic neuroimmunological mechanisms with excellent and thoughtful overviews on the genetic, molecular and general aspects of neuroimmunology. In the second section there are concise reviews on the immunopathological mechanisms of Multiple Sclerosis, noninflammatory immune mechanisms of CNS diseases and nervous system-immune system interactions. The third section covers monophasic autoimmune inflammatory diseases of the nervous system, chronic demyelinating diseases, tropical spastic paraparesis and the topical subject of HTLV-1 myelopathy, intraocular inflammatory disease and immune aspects of inflammatory myopathies.

I found the subsequent section on immunity and psychiatric disorders particularly interesting, covering the relationship between autoimmunity and depression, brain and behaviour and the immune system in general as well as neurological aspects of SLE. Non-inflammatory autoimmune diseases are then considered including very useful reviews of paraneoplastic syndromes, monoclonal gamopathies and neurological syndromes, disorders of the myoneural junction and motor neurone disease. The final chapters cover the subject of virus infection in $T$-cell deficient patients including SSPE, PML, a particularly thoughtful discussion of the pathogenesis of the AIDS dementia complex, behavioural correlates of HIV infection and finally the immunology of transmissible spongiform encephalopathy.

I found this book informative, stimulating, concise and full of original ideas. The production is excellent, the illustrations good and the referencing comprehensive. It is certainly not easy reading for the clinician without a working knowledge of neuroimmunology and it is also very expensive. Nevertheless, I shall find this volume very useful indeed and would certainly recommend that all good neurological and general medical libraries acquire it. Researchers in the field of neuroimmunology as well as many other clinicians and scientists would also find it to be a valuable aid to their work.

P G E KENNEDY

Cerebral Blood Flow and Metabolism Physiological Society Study Guides. BY A MURRAY HARPER AND SHEILA JENNETT. (Pp 110; Price: $£ 22.50$ hb; $£ 7.95$ pb). 1990, Manchester University Press. ISBN 0-71902892-2 hb, 2893-0 pb.

This concise, multi-author text is the latest in a series of Physiological Society Study Guides and is based on a teaching symposium on cerebral blood flow and metabolism, held in Glasgow in 1987, and updated to take into account recent developments. It is primarily aimed as an introductory text for those starting out in this increasingly complex field and is not intended as a research review for the seasoned investigator.

The five chapters: physiological control, innervation of the cerebral vasculature, cerebrovascular reactivity, measurement and anatomy of cerebral blood flow and the tomographic methods are clearly presented, with a useful review of important literature and supplemented by the frequent use of complementary figures. In this respect, the aims of the book have been achieved and I was 